\title{
LA INCIDENCIA DE LOS INCENDIOS FORESTALES EN EL PREPIRINEO OCCIDENTAL OSCENSE (1985-1994)
}

\author{
Fernando PÉREZ \\ Departamento de Geografía y Ordenación del territorio \\ Universidad de Zaragoza
}

\begin{abstract}
Resumen: Se cuantifica la incidencia de los fuegos forestales en el Prepirineo occidental oscense entre 1985 y 1994 y se analiza estadísticamente las causas y la distribución interanual. La gran incidencia de los fuegos forestales en el intervalo temporal considerado, especialmente en los años secos, y el importante porcentaje de hectáreas calcinadas como consecuencia de la caída de rayos, constituyen los resultados más significativos de este trabajo.
\end{abstract}

Palabras clave: Incendios forestales, rayo, negligencia, variabilidad interanual.

Abstract: This paper analyses the incidence of forest fires in the western Pyrinees of Huesca between 1985 and 1994, its causes and interannual distribution. The high frequency rate, specially during the dried seasons, its spatial distribution and number of burnt hectares caused by rays are the most significant results of the study.

Key words: Forest fires, ray, negligence, interannual variabiliy.

\section{INTRODUCCIÓN}

Aunque en el ámbito mediterráneo la dinámica del bosque está estrechamente ligada a las consecuencias derivadas de los incendios, la recurrencia y magnitud con que se están manifestando en las últimas décadas, está desestructurando, en ocasiones de manera irreversiblemente, los sistemas forestales afectados.

El caso español es especialmente grave ya que en las últimas décadas el fuego constituye la causa principal de deforestación. Con más de 10.000 incendios anuales y una superficie quemada —en algunos años- de más de 500.000 has ( $1 \%$ del territorio), España es uno de los países de la cuenca mediterránea más aquejados por este problema. 
A nivel provincial, Huesca no ha sido una de las más afectadas dentro del conjunto nacional, aunque dentro de su dominio forestal se pueden reconocer áreas especialmente proclives a sufrir este tipo de alteraciones medioambientales. El sector occidental de la zona Prepirenaica, tanto en términos de hectáreas calcinadas como en número incendios, constituye un buen ejemplo. La profusión de tormentas secas en verano, la gran extensión ocupada por plantaciones de coníferas y el incremento de la superficie dominada por formaciones subarbustivas, derivada del abandono de las actividades socio-económicas tradicionales explican, en buena parte, la fuerte incidencia del fuego en esta zona.

En este contexto, el objetivo de esta investigación es cuantificar la incidencia y gravedad del fuego en el Prepirineo occidental oscense, en el período comprendido entre 1985 y 1994, y analizar estadísticamente la causalidad del fuego y su distribución interanual.

Para ello se ha contado con los resímenes anuales de los incendios forestales de período considerado, facilitados por el Servicio Provincial de Montes de Huesca, y las memorias anuales sobre incendios forestales realizada por el Departamento de Agricultura y Montes de la Diputación General de Aragón (DGA), además de las aclaratorias aportaciones de los guardas forestales de la zona.

Este trabajo se incluye en otro de mayor envergadura denominado: «Estudio de procesos ambientales en zonas afectadas por fuegos forestales mediante datos de campo y técnicas de teledetección en el Prepirineo occidental oscense», cuyo objetivo principal es comprender y modelizar la dinámica ambiental posterior al incendio, y en donde el análisis de los incendios forestales desde las perspectivas señaladas, se erige como una de la fase iniciales de carácter prioritario.

\section{EL ÁREA DE ESTUDIO}

La zona de estudio es un territorio de aproximadamente $1.500 \mathrm{~km}^{2}-13.2387$ ha de superficie forestal-, inscrito en el complejo montañoso que constituyen las estribaciones meridionales del Sistema Pirenaico, en el ámbito correspondiente al sector occidental de la provincia de Huesca (Figura 1).

Básicamente se circunscribe a la cuenca media del río Gállego, en el tramo que va desde Sabiñánigo hasta Murillo, aunque también se extiende por otros sistemas fluviales como las cabeceras de los ríos Isuela, Flumen y Guatizalema, pertenecientes a la cuenca del Cinca-Segre y un tramo correspondiente a la margen izquierda del río Aragón a su paso por la Canal de Berdún.

En un contexto general típico de montaña media submediterránea, la zona presenta una enorme diversidad de ambientes topográficos, climáticos y fitogeográficos que se derivan de su singular situación geográfica, entre ambientes mediterráneos continentalizados por e] sur y atlánticos de montaña por el norte, y de su condición de zona de contacto entre los 


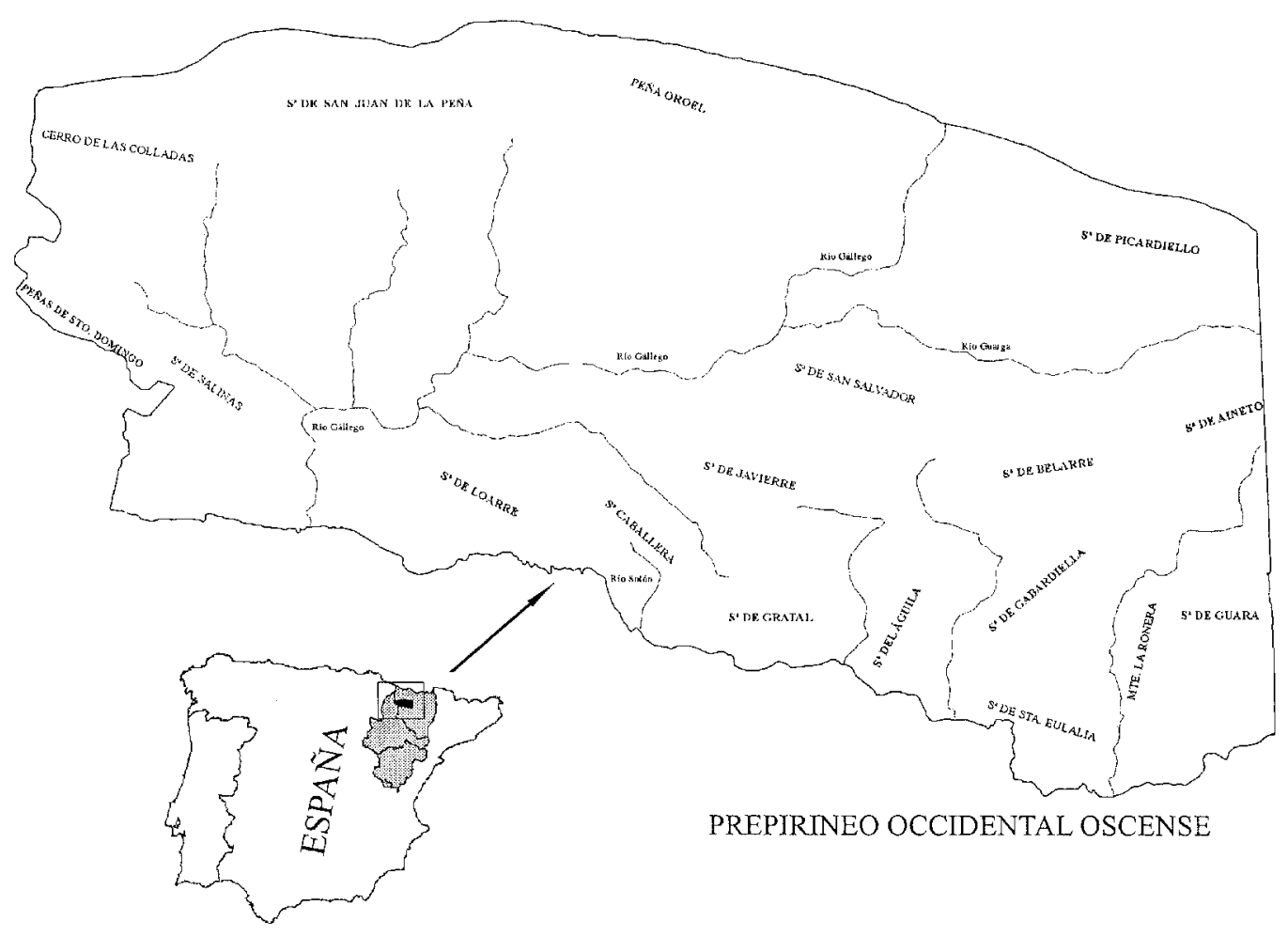

Figura 1. Ubicación del área de estudio

límites norte y sur de las regiones biogeográficas Mediterránea y Eurosiberiana respectivamente. Además la intervención del hombre a lo largo de la historia a terminado de modelar y recondicionar un espacio caracterizado por la presencia de vastas repoblaciones de Pinus sylvestris y Pinus nigra, quejigales y encinares naturales con distinto grado de madurez, formaciones subarbustivas y arbustivas pertenecientes a las alianzas Aphyllantion, Rosmarino-Ericion y Gypsophilion y algunos retazos de hayedo xerófilo y abetar.

\section{LA INCIDENCIA DE LOS INCENDIOS FORESTALES}

Entre 1985 y 1994, se han registrado un total 152 incendios forestales, que representan el $18,1 \%$ de los acontecidos en la provincia de Huesca en el mismo intervalo de tiempo (Tabla 1). El porcentaje se eleva al 51, $8 \%$ si en vez del número de incendios se considera el total de hectáreas quemadas, que en la zona que nos ocupa se cifra en 10.663-5.812 has arboladas y 4851 has no arboladas. 
Tabla 1. Número de incendios y hectáreas quemadas (1985-1994)

\begin{tabular}{|lcccc|}
\hline & & & \multicolumn{2}{c|}{$\%$ respecto a Huesca } \\
\cline { 4 - 5 } & $n .{ }^{\circ}$ de incendios & has afectadas & $\mathrm{n}^{\circ}$ de incendios & has afectadas \\
\hline Aragón & 2.539 & 67.619 & - & - \\
Huesca & 845 & 20.580 & $100 \%$ & $100 \%$ \\
Área de estudio & 152 & 10.663 & $51,7 \%$ & $17,9 \%$ \\
\hline
\end{tabular}

Fuente: D. G. A., elaboración propia

Además, de los 20 grandes incendios forestales - aquéllos que presentan un tamaño mayor de 500 has-ocurridos en la provincia de Huesca desde 1978, un total de 13 se han registraron en este sector. A la vista de los datos se puede constatar, la magnitud de la problemática del fuego en el Prepirineo occidental oscense, máxime si se tienen en cuenta que el área de estudio supone sólo el 14\% de la superficie forestal de Huesca.

Para valorar cuantitativamente la problemática del fuego en una zona, además de considerar y revisar las cifras absolutas, se han aplicado dos índices estadísticos que sintetizan algunos aspectos relacionados con este fenómeno: el índice de riesgo de incendios y el indice de gravedad de incendios. El primero expresa la frecuencia relativa de incendios y el segundo indica la importancia de éstos en el contexto forestal de una zona donde se apliquen (PADRÓ Y REINOSO, 1998).

$$
\begin{aligned}
& \text { índice de riesto de incendios }=\frac{\mathrm{n} .^{\circ} \text { de incendios }}{\text { superficie forestal }} * 10.0000 \\
& \text { indice de gravedad }=\frac{\text { superficie quemada }}{\text { superficie forestal }} * 100
\end{aligned}
$$

En Aragón, Huesca y el Prepirineo occidental oscense, la aplicación de estos índices ofrece resultados muy similares en el caso del indice de riesgo de incendios $(10,10$ y 11 respectivamente), pero en el caso del indice de gravedad esta paridad se desvanece debido a lo elevado del valor obtenido en el Prepirineo occidental oscense, si bien para Aragón y Huesca los resultados son bastantes similares (Tabla 2), de lo que se deriva que, los fuegos de la zona de estudio computan un nivel de gravedad relativo mucho más elevado que en el caso de Huesca y Aragón.

Tabla 2. Índices de riesgos y de gravedad en el periodo (1985-1994)

\begin{tabular}{|lcc|}
\hline & Índice de riesgo & Índice de gravedad \\
\hline Aragón & 10,1 & 2,7 \\
Huesca & 9,7 & 1,6 \\
Área de estudio & 11,4 & 15,5 \\
\hline
\end{tabular}

Fuente: D G. A., elaboración propia. 
Además, el 76,5\% de superficie quemada se relaciona con solamente el 4,6\% de los incendios, que es el porcentaje que representan los siete grandes incendios ( $>500 \mathrm{ha})$ registrados en la zona de estudio, y que son responsables del calcinamiento de 8.157 hectáreas (Tabla 3).

Entre las causas que se barajan para explicar este fenómeno se encuentra la alta inflamabilidad y combustibilidad ${ }^{1}$ de las formaciones vegetales dominantes — pinares naturales y repoblados y matorrales submediterráneos- y el mal estado de conservación de las mismas: grandes acumulaciones de vegetación arbustiva leñosa, espesura excesiva, etc. (PADRÓ Y REINOSO, 1998).

\section{ANÁLISIS DE LAS CAUSAS DE INCENDIOS}

El capítulo de las causas de los fuegos forestales en la zona de estudio está presidido por dos aspectos principales. En primer lugar, el alto número de incendios del que se desconoce la causa que los produjo, y en segundo lugar, el elevado porcentaje, tanto en número de incendios como en hectáreas quemadas que se deben a la caída de rayos $-41 \%$ y $42 \%$ respectivamente- (Figura 2). La profusión de tormentas secas en los meses estivales y la aleatoriedad del lugar de la recepción del rayo (zonas con grandes problemas de accesibilidad por cuestiones topográficas y de infraestructura), son las dos razones fundamentales que explicarían lo abultado de los guarismo en este sector. En lo que se refiere a los incendios intencionados destaca la enorme efectividad de estos, ya que, si bien en número no representan un porcentaje excesivamente elevado $-8 \%$-, en hectáreas calcinadas el porcentaje asciende hasta el $18 \%$ respecto al total.

Tabla 3. Tamaño y extensión de los incendios en el área de estudio

\begin{tabular}{|lcccc|}
\hline & & & \multicolumn{2}{c|}{$\%$ respecto al total de: } \\
\cline { 4 - 5 } & has afectadas & $n \cdot{ }^{\circ}$ de incendios & has afectadas & $n \cdot{ }^{\circ}$ de incendios \\
\hline$<1$ & 10 & 57 & 0,1 & 37,5 \\
$50-1$ & 667 & 76 & 6,3 & 50,0 \\
$500-51$ & 1.829 & 12 & 17,2 & 7,9 \\
$>500$ & 8.157 & 7 & 76,5 & 4,6 \\
Total & 10.663 & 152 & 100,0 & 100,0 \\
\hline
\end{tabular}

Fuente: D G. A., elaboración propia.

\footnotetext{
1 La inflamabilidad se define por el tiempo transcurrido hasta que se emiten gases inflamables bajo la acción de un foco de calor constante, mientras que la combustibilidad se refiere a la propagación del fuego dentro de una estructura vegetal (VÉLEZ, 1990).
} 

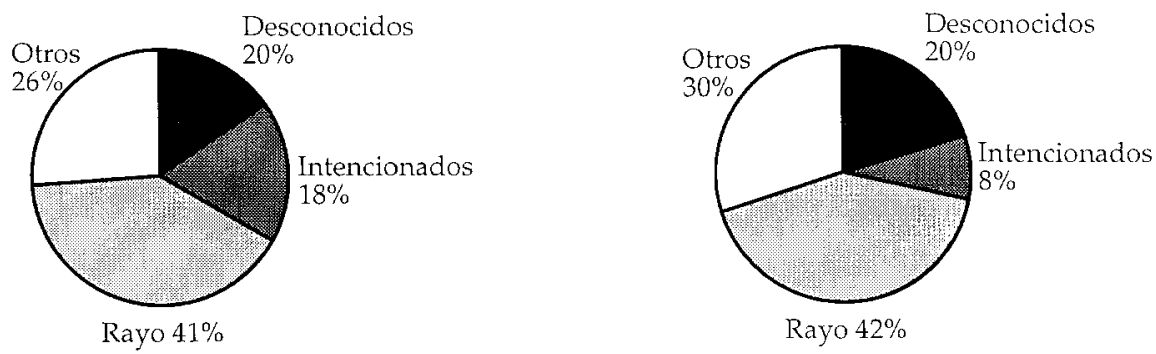

Figura 2. Porcentaje de hectáreas afectadas y de número de incendios según la causa (izda. hectáreas, dcha. número de incendios) Fuente: D G. A., claboración propia.

En el apartado de «otras causas» se han incluido todas aquellas relacionadas con aptitudes negligentes y otros motivos de carácter antrópico (quemas agrícolas incontroladas, maquinaria, maniobras militares, etc.). El número de incendios y el porcentaje de hectáreas calcinadas por estas causas también es considerable pero muestran una menor efectividad que en el caso de los intencionados ${ }^{2}$.

El análisis de las causas ha de complementarse con la caracterización de la distribución temporal de los fuegos, en este caso a lo largo de los diez años considerados, y su relación con las características meteorológicas de cada año en lo concerniente a los volúmenes precipitados en primavera y verano, toda vez que son las lluvias que más directamente se relacionan con la incidencia del fuego. Las primeras por cuanto contribuyen al desarrollo de las vegetación herbácea, y las segundas porque influyen en el estado fisiológico de las formaciones y en definitiva en la capacidad de combustión y propagación del fuego.

En este sentido, resulta enormemente significativa la fluctuación interanual que se registra. Más de tres cuartas parte de las hectáreas que ardieron lo hicieron en tan sólo tres años fatídicos: 1985, 1986 y 1994. La Figura 3 ilustra perfectamente este fenómeno, ya señalado por otros autores ${ }^{3}$.

Si relacionamos los datos anuales registrados en el área de estudio con los correspondientes a Huesca y Aragón, el carácter catastrófico de 1985 y 1986 resulta mucho más impactante. Lo que se quemó en el área de estudio en 1985 supuso el 71,6\% respecto al total provincial y el $46 \%$ en relación a lo quemado en Aragón. Todavía más alarmantes son los porcentajes del año 1986. En este caso las hectáreas quemadas supusieron el $86 \%$ y el

2 En este bloque ha de destacarse un incendio en particular, el que aconteció en la Sierra de San Juan de la Peña en 1994 que supuso el calcinamiento de cerca de 2000 has debido al descuido propiciado por un residente de Paternoy.

3 Según datos de Chauvelier (1990), la superficie total quemada en 1979 fue 603 has, 5.375 has en 1980 y 1.264 has en 1981, lo que da buena cuenta de la enorme variabilidad de este fenómeno. 


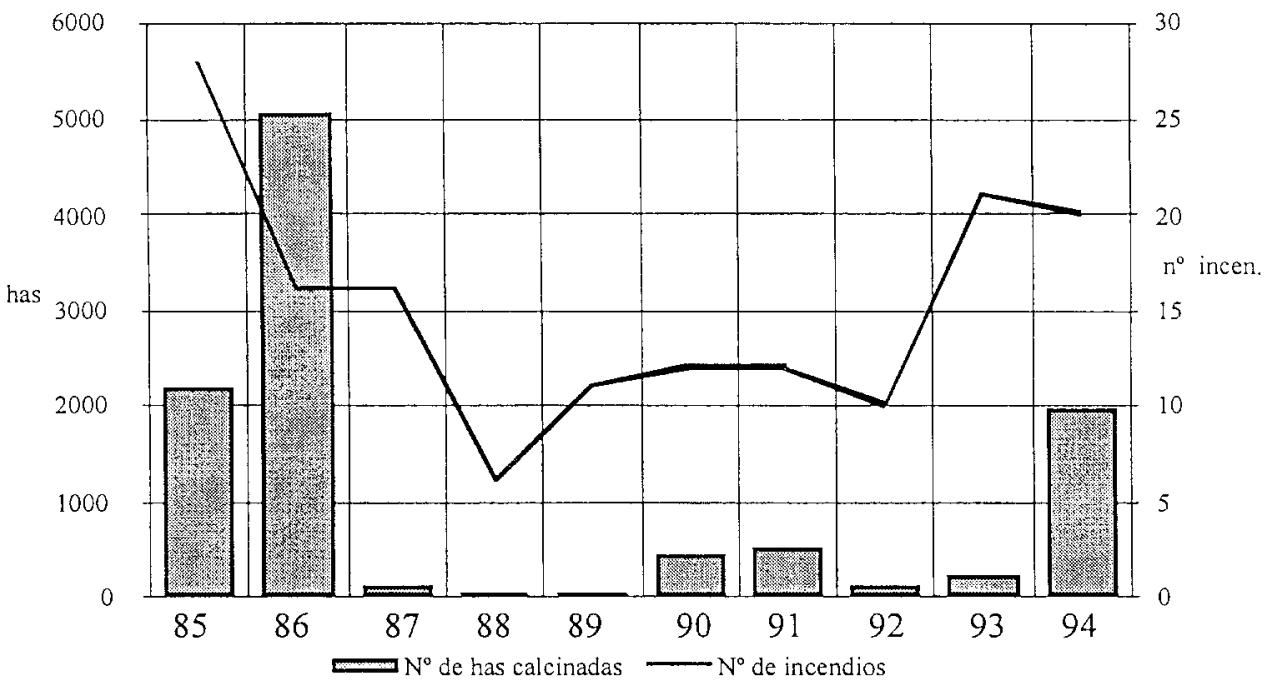

Figura 3. Distribución del número de hectáreas afectadas por año Fuente: D. G. A., elaboración propia.

Tabla 4. Distribución del número de incendios y de las hectáreas quemadas por año

\begin{tabular}{|lccccccccccc|}
\hline & 1985 & 1986 & 1987 & 1988 & 1989 & 1990 & 1991 & 1992 & 1993 & 1994 & Totales \\
\hline Has arboladas & 803 & 3.159 & 28 & 12 & 32 & 376 & 27 & 46 & 9 & 1.320 & 5.812 \\
Has no arboladas & 1.378 & 1.907 & 69 & 13 & 20 & 80 & 445 & 75 & 220 & 644 & 4851 \\
Has totales & 2.181 & 5.066 & 96 & 24 & 52 & 455 & 472 & 121 & 229 & 1.964 & 10.663 \\
N..$^{\circ}$ de incendios & 28 & 16 & 16 & 6 & 13 & 12 & 11 & 10 & 21 & 20 & 152 \\
\hline
\end{tabular}

Fuente: D. G. A., elaboración propia.

53,9 \% en relación a Huesca y Aragón respectivamente. En 1994 el porcentaje respecto a Huesca es también muy significativo, el $67 \%$, en cambio no lo es tanto respecto a Aragón, debido a la importante magnitud de los incendios que este año hubo también en las provincias de Teruel (incendio del Maestrazgo) y Zaragoza (incendio de las Altas Cinco Villas). En el otro extremo destaca lo reducido del número de hectáreas calcinadas en 1988 así como los porcentajes respecto a Huesca y Aragón 4,3\% y 1,2% respectivamente.

Al relacionar la incidencia interanual del fuego, en términos de superficie afectada, y el volumen de precipitación de cada año (Tabla 5), se observa un hecho lógico: los años en 
Tabla 5. Distribución anual de la precipitación de primavera y verano ${ }^{4}$

\begin{tabular}{|cccccc|}
\hline & \multicolumn{2}{c}{ Precipitación(mm) } & & \multicolumn{2}{c|}{ \% respecto a la media } \\
\cline { 2 - 3 } \cline { 5 - 6 } ANimavera & Verano & & Primavera & Verano \\
\hline 1985 & 208,03 & 71,53 & & 109,49 & 51,46 \\
1986 & 224,22 & 70,77 & & 118,01 & 50,91 \\
1987 & 143,59 & 145,12 & & 75,57 & 104,40 \\
1988 & 262,57 & 215,15 & & 138,20 & 154,78 \\
1989 & 173,54 & 126,65 & & 91,34 & 91,12 \\
1990 & 182,16 & 185,19 & & 95,87 & 133,23 \\
1991 & 140,61 & 68,98 & & 74,00 & 49,62 \\
1992 & 167,11 & 298,67 & 87,95 & 214,87 \\
1993 & 193,84 & 128,57 & & 102,02 & 92,50 \\
1994 & 119,62 & 42,07 & & 62,96 & 30,27 \\
media & 190,00 & 139,00 & & 100,00 & 100,00 \\
& & & & &
\end{tabular}

Fuente: I. N. M., elaboración propia.

los que más hectáreas se calcinan, los mencionados 1985, 1986 y 1994, son los años en los que se registran menos precipitación en verano (aproximadamente un 50\% menos de lo habitual), mientras que los años con menos superficie afectada $(1988,1990,1992)$ son los que presentan veranos más húmedos.

Desde el punto de vista del número de incendios esta relación no es tan acusada ya que existen años como 1987 y 1993 que, siendo años no excesivamente secos, presentan un gran número de incendios. Este hecho está relacionado con la importante cantidad de incendios declarados en primavera-invierno relacionados con actuaciones negligentes de carácter agrícola.

\section{CONCLUSIONES}

En líneas generales, del análisis de los incendios forestales del área de estudio podemos extraer las siguientes conclusiones:

En primer lugar que, en el contexto general de la provincia de Huesca, los incendios forestales registrados en el Prepirineo occidental oscense tienen un grado de incidencia espacial muy significativa, si bien en cuanto a número presenta niveles similares a los de la provincia de Huesca.

\footnotetext{
4 Los datos medios de precipitación han sido obtenidos a partir de 24 estaciones de registro climático comprendidas en el área de estudio. Los datos medios de precipitación (última fila de la tabla $n^{\circ} 4$ pertenecen a un período de 23 años - 1973 a 1995- (PÉREZ-CABELLO, 1999).
} 
En segundo lugar, que este tipo de manifestaciones irrumpen de manera irregular a lo largo del período considerado. Su acción devastadora, en términos de superficie, se concentra en años concretos, especialmente en aquéllos en los que las precipitaciones de verano representan la mitad o menos de las medias de esa estación.

En tercer lugar, que la causa principal que provoca la iniciación del fuego es de origen natural, el rayo, quedando en un segundo plano las causas relacionadas con acciones negligentes o intencionadas. Y que los incendios cuya causa se desconoce todavía presentan un porcentaje muy elevado.

En cuarto lugar, que los grandes niveles de devastación que adquieren algunos fuegos se deben, entre otras razones, a las características fisionómicas de las formaciones vegetales que configuran la mayor parte del complejo forestal de la zona de estudio y el pirofitismo que presentan las especies que las integran.

Destacan especialmente por su altos nivel de inflamabilidad y combustibilidad, las vastas repoblaciones de resinosas con carácter monoespecífico, carentes de tratamientos silvícolas apropiados, donde se acumulan grandes cantidades de combustible, y las comunidades arbustivas y subarbustivas, cuya extensión se ha incrementado enormemente en la última mitad de siglo como consecuencia del abandono de las actividades tradicionales a partir de los 50. En todas ellas el fuego encuentra unas enormes facilidades para propagarse con rapidez cuando irrumpe de manera incontrolada.

\section{AGRADECIMIENTOS}

Este trabajo ha sido posible gracias al apoyo financiero de la Diputación General de Aragón, del Instituto de Estudios Altoaragoneses y de la Universidad de Zaragoza.

\section{BIBLIOGRAFÍA}

CHAUVELIER, F. (1990): La repoblación forestal en la provincia de Huesca y sus impactos geográficos. Colección de Estudios Altoaragoneses, 34. Huesca.

PADRÓ, A. y REINOSO, J. (1998): «La campaña de prevención de incendios forestales, a punto», Surcos de Aragón, 46, 32-36. Dpto. de Agricultura y Medio-Ambiente. Gobierno de Aragón. Zaragoza.

PÉREZ-CABELLO, F. (1999): La dinámica ambiental posterior al incendio forestal: Modelización en el Prepirineo occidental oscense. Serie: Microfichas, Prensas Universitarias de Zaragoza. Zaragoza. 213 pp.

VÉLEZ, R. (1990): «Algunas observaciones para una selvicultura preventiva de incendios forestales». Ecologín, 1, 561-571. Madrid. 